Elsevier Editorial System(tm) for Best

Practice \& Research Clinical Obstetrics \& Gynecology

Manuscript Draft

Manuscript Number: YBEOG-D-20-963

Title: A comparison of recent guidelines in the diagnostic and management of placenta accreta spectrum disorders

Article Type: Issue on PASD (GE_EC)

Corresponding Author: Professor Eric Jauniaux, MD, PhD, FRCOG

Corresponding Author's Institution: Royal Free and University College London

First Author: Eric Jauniaux, MD, PhD, FRCOG

Order of Authors: Eric Jauniaux, MD, PhD, FRCOG; John Kingdom 

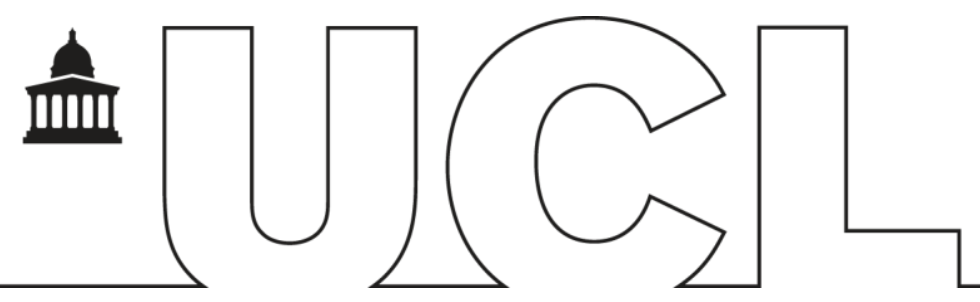

London $29^{\text {th }}$ May 2020

The Editor

BP\&R in Clin Ob Gyn

Dear Edwin,

Please find enclosed our manuscript entitled "A comparison of recent guidelines in the diagnostic and management of placenta accreta spectrum disorders" and the corresponding MCQs.

The final version has been approved by all authors who have equally contributed to the development of this project and have no conflict of interest to declare.

The length of the manuscript including the main text, references and the 2 tables is around 7000 words as required.

Very best regards

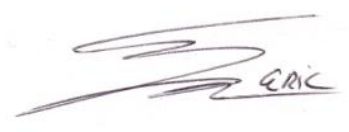

Eric Jauniaux 
*Highlights (for review)
Highlights:

*Highlights (for review)
Highlights:

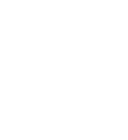

ghights:

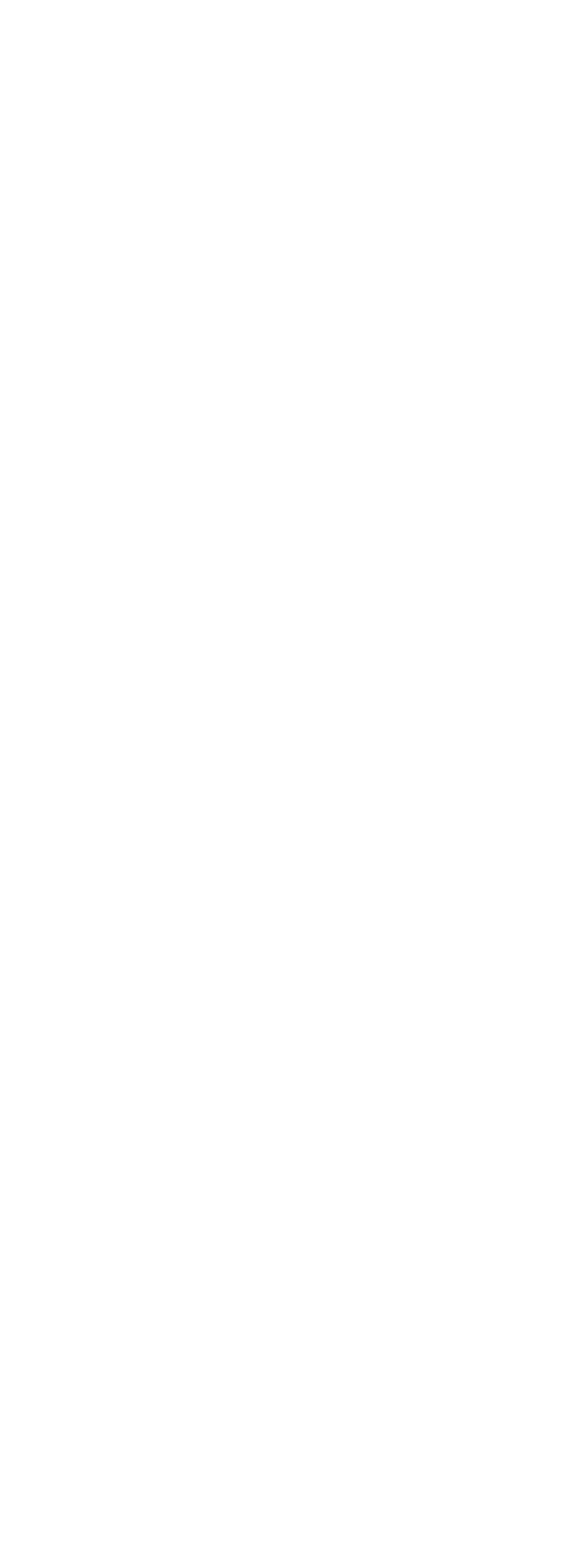




\title{
A comparison of recent guidelines in the diagnostic and management of placenta accreta spectrum disorders
}

\author{
Eric Jauniaux ${ }^{1}$, John Kingdom ${ }^{2}$, Robert M. Silver ${ }^{3}$,
}

${ }^{1}$ EGA Institute for Women's Health, Faculty of Population Health Sciences, University College London (UCL), London, UK.

${ }^{2}$ Department of Obstetrics and Gynecology, Mount Sinai Hospital, University of Toronto, Toronto, ON, Canada.

${ }^{3}$ Department of Obstetrics and Gynecology, Mount Sinai Hospital, University of Toronto, Toronto, ON, Canada

The authors report no conflict of interest.

The views expressed in this document reflect the opinion of the individuals and not necessarily those of the institutions that they represent.

Word count Text \& references: 6444

Corresponding author: Professor Eric Jauniaux, EGA Institute for Women's Health, University College London, 86-96 Chenies Mews, London WC1E 6HX, UK.

Telephone numbers: $+44 / 207 / 3908113$

Fax: $+44 / 207 / 3908115$

E-mail: e.jauniaux@ucl.ac.uk

\section{ABSTRACT (max 150 words)}


Accreta placentation and in particular its invasive forms are impacting maternal health outcomes globally and the prevalence of placenta accreta spectrum (PAS) continues to increase. The Royal College of Obstetricians and Gynaecologists (RCOG) and the American College of Obstetricians and Gynecologists (ACOG) with the Society for Maternal-Fetal Medicine (SMFM) have updated their national guidelines whereas the Federation International of Gynecology and Obstetrics (FIGO) and the Society of Obstetricians and Gynecologists of Canada (SOGC) have developed new guidelines on the diagnosis and management PAS. A comparison of these guidelines highlights common strong recommendations on the need to carefully evaluate women at high-risk for PAS (e.g. prior uterine surgery presenting with anterior low-lying placenta or placenta previa), using multi-modal ultrasound imaging. For women diagnosed with PAS, multidisciplinary team-based care, with full logistic support structures (immediate access to comprehensive blood products, adult and neonatal intensive care) and established expertise in complex pelvic surgery, is critical to maximize safe outcomes for mother and newborn.

Key Words: Placenta accreta; increta; percreta; prenatal diagnosis, risk factors, conservative management, surgical management, guidelines. 


\section{BACKGROUND}

\section{A. Historical perspective}

The first fully documented cases of birth complicated by accreta placentation were published almost one hundred years ago [1-4]. In 1937, Irving and Hertig published a cohort of 18 cases with similar clinical and pathological observations, characterized by abnormal mechanical adherence of the placenta to the underlying uterine wall, associated with severe post-partum hemorrhage $(\mathrm{PPH})$, and requiring a hysterectomy in a majority of 15 cases [5]. Histologic observations noted partial or complete absence of the decidual layer which is normally present between the anchoring placental villi and the superficial myometrium. Although this series did not include a single case of placental tissue invading beyond the uterine wall, these seminal observations by Irving and Hertig remain in use today as key criteria for the diagnosis of both adherent and invasive placentation. However, they are often applied to describe the retained placenta as also being pathologically-adherent to the uterine wall [6-10]. This trend has subsequently led to increasing, and highly variable, reported rates in the overall prevalence of placenta accreta, ranging between $0.01 \%$ and $1 \%$ of livebirths $[11,12]$.

Lukes et al. [13], in 1966 were the first group to propose a detailed histologic classification for placenta accreta based on the depth of the villous penetration of the myometrium; starting with placenta adherenta or creta (PC) when the villi adhere directly to the myometrium without a decidual interface. Further invasion was defined as placenta increta $(\mathrm{PI})$ when the villi invade into the myometrium. Finally, placenta percreta (PP) occurs when the villi invade the full thickness of the uterine wall either to the serosa or beyond to involve extra-uterine structures, such as the bladder. They also observed that penetration of the myometrium by placental villi is rarely a 
uniform process, such that areas of normal villi attached to decidua, adherent villi attached to myometrium, and invasive villi deeper within the myometrium, may all coexist within the same specimen. To address this constellation of variable findings, a majority of experts use the term "placenta accreta spectrum" (PAS). This term includes all grades of abnormal placentation and is used as the basis for the development of a new clinical classification proposed by the Federation International of Gynecology and Obstetrics (FIGO) [14].

Many clinicians and the World Health Organization (WHO) international classification of diseases (www.who.int/classifications/icd) continue to use the 1937 Irving and Hertig definition for placenta accreta and therefore make no distinction between different grades of PAS. Only $10 \%$ of studies on the prenatal diagnosis of PAS and placenta previa accreta provide detailed histopathology data on the different grades of PAS, and the corresponding distribution varies widely (Table 1). In addition, many authors and the WHO are adding to the confusion by using a $19^{\text {th }}$ century "morbidly adherent placenta" terminology which refers only to abnormally adherent PAS. This has led to many authors to over-diagnose the incidence of PAS by including cases which present as complete or partial placental retention, or with excessive bleeding from the placental bed at Cesarean delivery $[6,8,10]$. Furthermore, in women delivered by repeat Cesarean, there may be a tendency to over-diagnose PAS in circumstances where the low-lying anterior placenta is visible through the old thin scar region due to lower-segment dehiscence. This scar may expand during pregnancy to create a large uterine "window" through which a portion of the placenta is visible - without any villous tissue truly invading the serosa and/or the surrounding myometrium [15]. 
A new standardised classification and reporting guidelines for the pathology diagnosis of PAS has also been recently proposed by an expert panel [16]. This new classification, together with the 2016 standardized ultrasound and MRI descriptions and proforma reporting for suspected antenatally with placenta accreta, [17-19] have been established with the intent of improving the overall quality of epidemiologic data on PAS incidence. This system also may improve management outcome data, by allowing stratification using the standardized different grades of PAS.

\section{B. Epidemiology}

Until the 1950s, the predisposing factors for PAS in subsequent pregnancies were manual removal of the placenta, endometritis and/or "vigorous" uterine curettage during a prior delivery [5]. Today, over $95 \%$ of women diagnosed prenatally with PAS present with a low-lying/placenta previa and a surgical history of at least one prior uterine surgery, most commonly Cesarean delivery (CD) [20-22]. The rapid increase in $C D$ rates around the world, together with a greater prevalence of prior gynecologic surgery (myomectomy, uterine adhesiolysis, septal resection), means that up to $6 \%$ of the pregnant population have the combination of a low-lying anterior placenta and prior uterine surgery, at their 12-week nuchal translucency ultrasound examination (Panaitova et al., USOG 2018). Advanced reproductive age at conception and use of in-vitro fertilization methods have also contributed to the increased incidence of placenta previa. For example, there is strong epidemiologic evidence that the incidence of placenta previa accreta increases with the number of prior CDs (22-24). The ratio of adherent/invasive accreta placentas has changed from $7 / 3$ in the 1970 s to $5 / 5$ in the last two decades [22], a change that is directly linked to the increase in the number of grand multiparas with multiple $C D$ scars undertaking further 
pregnancies $[25,26]$. Pregnancy following multiple CDs is also a major risk factor for large cesarean scar defects (CSD), and for the development of cesarean scar pregnancies (CSP) in the cervico-isthmic niche created by focal myometrial loss at the site of prior CDs [27]. The diagnosis of CSP in the first trimester is increasingly common, such that the natural history of evolution to PAS has now been clearly established, especially for type-2 CSP. Since CSPs may lead to early uterine rupture and emergency hysterectomy [28], or more increasingly, be managed immediately by medical, or laparoscopic surgery methods (PMID 28601471), their diagnosis and management will further alter the epidemiology of PAS at birth.

PAS is not exclusively a consequence of $C D$ and has been reported in primiparous women with a history of operative hysteroscopy, suction curettage, surgical termination, endometrial ablation, uterine pathology such as bicornuate uterus, adhesiolysis for Asherman's syndrome, adenomyosis, endometrial resection, polyvinyl bead embolization of fibroids, and myotonic dystrophy [22,25,29]. In fact, any procedure causing surgical or substantial and chronic damage to the integrity of the uterine wall, especially if the endometrium fails to re-epithelize in an area of scar formation, has been associated with PAS $[25,26]$. Accreta placentation can occur after myomectomy but the risk is low [30] unless the uterine cavity is entered, because the endoscopic removal of intramural or sub-serous fibroids does not involve the uterine cavity [31]. Though the removal of fibroids in the upper part of the uterus is less likely to lead to uterine dehiscence [32], focal PAS may still occur. Undoubtedly PAS has increased as a consequence of modern obstetric and reproductive medical practices, and has been accentuated by decisions to delay childbearing, the need for subsequent reproductive assistance and the prevalence of prior gynecologic surgery [25]. 


\section{The "World No 1" surgical procedure effect}

Safe Caesarean delivery (CD) is an essential component of a secure, functioning and comprehensive maternity system, and is now the most commonly performed major operation around the world, with more than 1 million procedures performed each year in the USA alone [33]. In 1985, a group of experts advising the WHO highlighted an unjustified and remarkable increase in $C D$ rates worldwide in the prior decade [34]. They stated there was no justification for any country or region to have a caesarean section rate higher than $10-15 \%$. This recommendation has had little impact on the rates of CD in most countries of the world, which have continued to rise over the following four decades [35]. In 2011, Solheim et al., published a decision-analytic model using data on national birthing order trends after CD rates in the U.S. between 1995 and 2005. They estimated that if the number of primary and secondary CDs continue to rise, by 2020 the CD rate will be $56.2 \%$, and that as a consequence there will be an additional 6236 placenta praevias, 4504 PAS, and 130 maternal deaths annually [36]. The CD rates have not risen to this level in the U.S. but have risen above $40 \%$ over the past decade in many middle-income countries around the world with mixed public-private health care systems, such as Brazil, Turkey, Italy, Egypt, Argentina, Iran, South Korea and Mexico. Solheim et al., calculated that the rise in CD-associated complications will lag behind the rise in CDs by around 6 years [36]. These data highlight the need for local health providers to become prepared for a significant rise in the numbers of pregnancies complicated by placenta previa accreta. In particular, in middle-income countries with high-birth rates such as Turkey and Egypt, where more than half of all births are via CD, the 
prevalence and negative impacts of PAS have now outweighed the benefits of improved access to quality obstetric care at the population-based level.

The 2017 report from the UK and Ireland Confidential Enquiries into Maternal Deaths indicated that although there was no significant change in the maternal death rate in the UK between 2010-12 and 2013-15, an increase in the number of deaths associated with PAS was observed [37]. Not surprisingly, as PAS has become a new risk factor for major obstetric complications, there has been an exponential increase in the number of articles published in the medical literature over the last decade. In an editorial for the special issue of the International Journal in Gynecology and Obstetrics we reported that a PubMed (www.ncbi.nlm.nih.gov/pubmed) search of the term "placenta accreta" up to $1^{\text {st }}$ of December 2017 generated 2296 hits [20]. Through the $1^{\text {st }}$ of May 2020, there have been another 569 new articles published. Each year since 2010, more publications on placenta accreta have occurred than during the entire period between 1947-1962. These new data have stimulated the development of new national and international guidelines and the updating of previous guidelines. These guidelines have extracted, analyzed and summarized recent quality evidence-based data on diagnosis and management of PAS. They are therefore essential to inform local and international policies and health provision for the development of maternity centres with specialist surgeons, equipment, drugs, blood bank and intensive care infrastructure to safely manage the new epidemy of women presenting with PAS.

\section{CLINICAL GUIDELINES FOR DIAGNOSIS AND MAGEMENT OF PAS}

\section{A. New and updated guidelines}


There are currently two recently updated and two new guidelines on the diagnosis and management of PAS available in the recent international medical literature:

- The 2018 Royal College of Obstetricians and Gynaecologists (RCOG) Greentop Guideline No. 27a which includes both placenta praevia and placenta accreta and has replaced the 2011 edition of the guidelines Placenta Praevia, Placenta Praevia Accreta and Vasa Praevia: Diagnosis and Management. This is the $4^{\text {th }}$ edition of this guidelines and the first to be published in the British Journal of Obstetrics and Gynaecology [38]. The first edition, published in 2001, was entitled Placenta Praevia: Diagnosis and thus did not include placenta accreta.

- The 2018 American College of Obstetricians and Gynecologists (ACOG) obstetric care consensus was developed jointly with the Society for MaternalFetal Medicine (SMFM) and endorsed by the Society of Gynecologic Oncology (SGO) and replaced the Committee Opinion No. 529, of July 2012 and SMFM Clinical Guideline of November 2010. These guidelines were published simultaneously in Obstetrics and Gynecology [39], the official journal of ACOG and the American Journal of Obstetrics and Gynecology (40).

- The 2018 FIGO consensus guidelines on placenta accreta spectrum disorders were developed by the Safe Motherhood and Newborn Health Committee [41]. The guidelines are presented in four separated expert reviews: Epidemiology [22], prenatal diagnosis and screening [42], non-conservative surgical management [43] and conservative management [44] and were published simultaneously on the FIGO website and a special issue of the 
International Journal of Gynaecology and Obstetrics, the official journal of the FIGO.

- $\quad$ The 2019 Society of Obstetricians and Gynecologists of Canada (SOGC) No. 383-screening, diagnosis, and management of placenta accreta spectrum disorders [45] which was published simultaneously in English and French in the Journal of Obstetrics and Gynaecology of Canada, the official journal of the SOGC.

\section{B. Development process}

- The RCOG guidelines are developed by stakeholders representing heath care in the UK from the best research evidence. They are recommendations for good practice that are not meant to dictate care, but rather to support healthcare professionals in their work; some recommendations may not be transferable to non-UK settings. The guideline authors included a lead developer and a team of local and international multidisciplinary group of experts who assessed the evidence gathered in a systematic matter. The authors evaluated the methodology of each study and graded the evidence from level 1 to level 4 depending on its individual value for the guidelines. The guideline authors drew up recommendations for each point, which are graded A, B, C and "good practice" according to the strength of the evidence that supports them (Table 2). The draft guideline document then went through a process of consultation and peer-review and the final version was approved by the Guidelines Committee and the RCOG Clinical Quality Board. In addition to the guidelines, the RCOG produced an information leaflet for the public. 
- The ACOG and SMFM guidelines are developed in a fashion quite similar to the RCOG document. They are also considered recommendations or guidelines, with the recognition that not all clinicians and settings have equivalent resources. The guideline authors included a lead developer and a national team of maternal-fetal-medicine based experts with representation from both societies. The authors were provided a compilation of available studies and data that were gathered in a systematic matter. The authors evaluated the methodology of each study and used them to make recommendations. Recommendations were given as strong (1), weak (2), or best practices (in the absence of evidence). In addition, the quality of evidence was graded as A (high), B (moderate) and C (low) (Table 3). The draft guideline document then went through a process of consultation and peer-review and the final version was approved by the ACOG Obstetric Guidelines Committee and the SMFM publications committee.

- The FIGO guidelines: were developed as expert evidence-based opinions with the aim of helping clinicians, policymakers, and patients to make wellconsidered decisions about health care for a particular condition. The guidelines were intended to be transferable to an international setting and were therefore based on consensus from a group of international specialists who were individually proposed for their expertise by their national professional societies of obstetricians and gynaecologists. The guidelines provided clear, well-reasoned information such that decision makers can use them effectively both nationally and globally. The quality of evidence was graded as high, medium or low according to the methodology of the studies 
reviewed. The guideline authors drew up recommendations which were graded as strong or weak and combined these into a table with the quality of evidence. The final version was approved by the FIGO Safe Motherhood and Newborn Health Committee.

- The SOGC guidelines: were developed by the multidisciplinary team members based at the largest site providing care for women with PAS in Canada with prior approval by the national society SOGC. The final guideline was approved by both the maternal-fetal medicine and the diagnostic imaging guideline committees using the established evidence-based grading systems used for all SOGC national clinical practice guidelines. Broad consensus was achieved across Canada as reflected by the final author listing. Members from this guideline also contributed to the FIGO guidelines.

\section{COMPARISON OF THE RCOG, ACOG/SMFM, FIGO AND SOCG GUIDELINES}

The classification of evidence levels and recommendations provided by the different guidelines is directly linked to the quality of the data available in studies published at the time of the development of the corresponding guidelines. The recommendations therefore changed to some extent with time, as is illustrated by the RCOG and ACOG/SMFM guidelines, which are now in their $3^{\text {rd }}$ editions. In the case of PAS, most of the cohort studies published before 2015 are retrospective and thus lack the more accurate modern clinical descriptions and detailed histopathologic examinations required to confirm the diagnosis of the different grades of PAS, and therefore their specific management recommendations. Recent systematic reviews 
and meta-analyses on the overall prevalence of PAS and the incidence of placenta previa accreta show large amounts of heterogeneity between population studies for the prevalence, incidence and distribution of the different grades PAS, as well as rates of peripartum hysterectomy, haemorrhage requiring transfusions and maternal mortality. These wide variations between studies are due to inconsistency with regards to the criteria used to diagnose and confirm the nature of PAS at birth $[12,21]$. Surprisingly there is also high heterogeneity in qualitative and diagnostic data on the ultrasound criteria used in cohort studies, reporting on placenta previa accreta, for the much simpler ultrasound diagnosis of placenta previa and the gestational at confirmation of the diagnosis [21].

\section{A. On prenatal diagnosis}

A 2017-2018 international survey of practices used in the diagnosis and management of PAS disorders found that $92 \%$ of respondents indicated that their department routinely performs prenatal screening of women identified as being at high risk of PAS disorder [46]. Of those, a similar \% reported that such screening was prompted by a prior history of uterine surgery and/or cesarean delivery plus a low-lying placenta or placenta previa at mid-pregnancy using grey-scale transabdominal ultrasonography. The most common additional method of diagnosis among women with a high suspicion for PAS disorders was transvaginal sonography (TVS) and over $61 \%$ use both ultrasonography and magnetic resonance imaging (MRI).

All the guidelines agree that:

- Antenatal diagnosis of PAS is crucial in planning its management and has been shown to reduce maternal morbidity and mortality. 
- Ultrasonography is a relatively inexpensive and widely available imaging

- Previous CD and the presence of an anterior low-lying/placenta praevia should alert the antenatal care team of the higher risk of PAS.

- MRI is not essential for making a prenatal diagnosis of suspected PAS and is therefore not the preferred primary imaging modality for the initial evaluation of women at risk of PAS.

The RCOG guidelines identify ultrasound imaging as highly accurate (> 95\%) when performed by a skilled operator with experience in diagnosing PAS (Grade C) and the ACOG/SMFM highlights that although ultrasound evaluation is important, the absence of ultrasound findings does not preclude a diagnosis of PAS and that clinical risk factors remain equally important as predictors of PAS by ultrasound findings (Grade $1 \mathrm{~A}$ ). In addition, the SOGC states that the effectiveness of ultrasound in the context of PAS depends upon awareness of clinical risk factors, imaging quality, operator experience, gestational age, imaging modalities, and adequate bladder filling. The FIGO strongly recommends that the ultrasound signs of PAS should be described using standardised protocols. On the basis of at least one systematic review and meta-analysis, the RCOG guidelines recommend referral of women with any ultrasound features suggestive of PAS to a specialist unit with imaging expertise (Grade B). The ACOG/SMFM suggest performance of ultrasound examinations at approximately $18-20,28-30$, and $32-34$ weeks of gestation in asymptomatic patients. The RCOG indicates that TVS has an important role in the early diagnosis, follow-up, differential diagnosis between adherent and invasive accreta placentation and overall management of PAS [Evidence level 4].

For the RCOG, FIGO and SOGC, MRI may be useful in evaluating the pelvic 
extension of a placenta percreta or areas difficult to evaluate on ultrasound, though its effectiveness is currently limited by the relative contraindication to the use of a gadolinium contrast enhancing agent. For ACOG it is unclear whether MRI improves diagnosis of PAS beyond that achieved with ultrasonography alone. On the basis of two systematic reviews and meta-analyses, the RCOG concluded that the diagnostic value of ultrasound imaging and $\mathrm{MRI}$ in detecting PAS is similar (Grade C). Furthermore, the focus and experience of the supervising/reporting radiologist remains an important and less well-studied factor in the diagnostic accuracy of MRI, since access to expert radiologists with a special interest in pregnancy is highly variable between centers.

\section{B. On preparing for delivery}

A recent anonymous survey of the 154 obstetrical services in NHS England found that of the 114 that responded, $70 \%$ manage their PAS cases "in-house", despite one third of these units reporting that they manage on average only one case per year [47]. The 23 units that describe themselves as "specialist centres" managed a median of four cases per year. There is mounting evidence from before and after the publications of the new guidelines that women with PAS diagnosed prenatally and managed by a multidisciplinary team (MDT) in a centre of excellence (CoE) are less likely to require emergency surgery, large-volume blood transfusion and reoperation within 7 days of delivery for bleeding complications compared with women managed by standard obstetric care without a specific protocol [48]. Although, there is no evidence for an ideal minimal number of cases of PAS managed per month or year, maternal outcomes are improved over time with increasing experience within a wellestablished MDT performing 2-3 cases per month [49,50]. 
There are also wide variations between healthcare providers within the U.S. $[51,52]$ and between different countries $[46,53]$ in the preparation for the surgical procedure such as the use of cystoscopy and placement of ureteral stents, the involvement of different surgical specialities in the operating team, the choice of anaesthesia technique and the use of additional techniques such as interventional radiology (IR) pre-operative placement of an arterial balloon either in the lower aorta, common iliac or anterior divisions of the internal iliac arteries.

All the guidelines recommend that:

- Patients diagnosed with PAS should be cared for by a MDT with expertise in complex pelvic surgery in a specialist centre with logistic support for immediate access to blood products, adult and neonatal intensive care.

- Preoperative coordination with protocol-based interdisciplinary care including anesthesiology, hematology/blood bank, maternal-fetal medicine, neonatology, and expert pelvic surgeons to optimize intraoperative and postoperative outcomes.

- Preoperative counselling should include review of planned and possible alternate surgical strategies and complications and that a contingency plan for emergency delivery should be developed, including the use of an institutional protocol for the management of maternal haemorrhage.

All the guidelines agree that:

- Use of antenatal corticosteroids for lung maturation by 32-34 weeks is appropriate in women with antenatally diagnosed PAS and with current 
national and international gestational age-based recommendations for anticipated delivery before term.

- No amniocentesis is necessary because data regarding pulmonary maturity do not change clinical recommendations for emergency delivery before 36 weeks.

- There are currently insufficient data to recommend the routine use of cystoscopic assessment of the bladder and placement of ureteric stents but collaboration with a urologic surgeon is advisable in cases with suspected bladder wall involvement.

- Optimizing hemoglobin values during pregnancy is important and when iron deficiency is noted oral replacement or intravenous infusions should be implemented and when available intraoperative cell salvage may be utilized or be on "stand-by".

- Timing of delivery decisions need to balance maternal risks and benefits with those of the fetus or neonate.

The guidelines give different opinions for the optimal timing for delivery. For the ACOG/SMFM between $34^{+0}$ and $35^{+6}$ weeks of gestation is the recommended gestational age for scheduled cesarean delivery or hysterectomy absent extenuating circumstances in a stable patient $(1 \mathrm{~A})$ whereas for the RCOG, in the absence of risk factors for preterm delivery and/or bleeding from a placenta previa, indicate that planned delivery at $35^{+0}-36^{+6}$ weeks of gestation provides the best balance between fetal maturity and the risk of unscheduled delivery [GPP]. The SOGC recommends that for otherwise healthy women with no history of vaginal bleeding, the optimal timing of elective CD is around 34-36 weeks of gestation (II-3B). FIGO guidelines are based on consensus from expert from high-, medium- and low-resources 
countries. Low resource countries, in particular in Africa have often limited or no access to blood products and ICU and thus the FIGO does not formally recommend a preferred time for delivery as for low resources countries moving the delivery date as close as possible to 37 weeks has a direct impact in reducing poor neonatal outcome.

A survey of 26 Israeli hospital maternities reported that general anesthesia was used almost exclusively among women with high suspicion for PAS disorders [53]. The international survey of FIGO experts found that spinal-epidural anesthesia was used by $44 \%$ of the respondents [46]. The RCOG recommends that the choice of anaesthesia technique should be should be made by the anaesthetist conducting the procedure in consultation with the patient in advance [GPP] whereas for the SOGC, regional epidural anaesthesia is considered safer than general anaesthesia in most instances, is associated with reduced blood loss, is preferred by patients and their partners and affords a 24-hour window of effective post-operative pain relief (II-2A). The RCOG also recommend that the patient should be informed that the surgical procedure can be performed safely with regional anaesthesia but should be advised that it may be necessary to convert to general anaesthesia if required and asked to consent [D]. The ACOG/SMFM does not comment on this issue and the FIGO guidelines highlight that there is insufficient evidence to support the use of one technique over the other.

\section{On management}

Overall four management approaches have been described alone or in combination with additional procedures mainly iliac or uterine artery devascularisation techniques, either surgical or using interventional radiology (IR). 
The radical techniques are:

- Primary hysterectomy $(\mathrm{PH})$ following immediately the delivery of the infant, without attempting placental separation.

- Delayed or secondary hysterectomy between 3 days and 12 weeks after delivery of the infant, leaving the placenta in situ, with repair of the hysterotomy incision.

The conservative techniques are:

- Leaving partially or totally the placenta in situ after delivery of the infant, avoiding the placenta, with repair of the hysterotomy incision (expectant management).

- Partial myometrial resection (partial excision of the accreta area) following the delivery of the infant, and without disturbing the placenta, followed by repair of the uterus.

Planned preterm caesarean hysterectomy with the placenta left in situ has been the recommended management strategy for PAS by both RCOG and ACOG/SMFM in the previous version of their guidelines. Not surprisingly, surveys of healthcare providers in the U.S. published in 2012 [51] and 2013 [52] found that most ACOG fellows SMFM members proceeded with hysterectomy and only $14.9 \%-32.0 \%$ reported attempting conservative management. The 2018 international survey reported that around $60 \%$ of the respondents favored a radical surgical management approach with cesarean hysterectomy i.e. primary cesarean hysterectomy with the placenta left in situ, whereas $10(28 \%)$ would perform a partial myometrial resection or radical dissection whenever possible [46]. Primary attempt at placental removal and compression sutures were attempted by a quarter of experts and around half of the respondents used intra-arterial balloons or arterial embolization [46]. 
All the guidelines recommend that:

- The delivery hysterotomy should be performed high without incising through the placenta.

- No attempt should be made to remove the placenta (extirpative approach or forcible manual removal of the placenta) if it shows no signs of separation as this may cause substantial hemorrhage.

- If at the time of an elective repeat caesarean section, PAS is suspected based on uterine appearance and there are no extenuating circumstances mandating immediate delivery, the caesarean section should be delayed until until optimal surgical expertise arrives and adequate blood products are available.

- Conservative management or expectant management should be considered only for carefully selected cases of PAS after detailed counseling about the risks, uncertain benefits, and efficacy and local arrangements need to be made to ensure regular review, ultrasound examination and access to emergency care should the patient experience complications.

- Uterus-preserving surgical techniques should only be attempted by surgeons working in teams with appropriate expertise to manage such cases.

- MTX adjuvant therapy should not be used for expectant management as it is of unproven benefit and has significant adverse effects, including maternal mortality.

All the guidelines agree that: 
- There is insufficient evidence to recommend giving or withholding uterotonic drugs after delivery of the fetus

- There is insufficient evidence to recommend routine IR techniques such as embolisation or placement of an arterial segment balloon designed to arrest arterial blood flow that may supply the gravid uterus.

The RCOG guidelines indicate that when the extent of the placenta accreta is limited in depth and surface area, and the entire placental implantation area is accessible and visualised (i.e. completely anterior, fundal or posterior without deep pelvic invasion), uterus-preserving surgery may be appropriate, including partial myometrial resection. [GPP]. The ACOG/SMFM guidelines highlight that because of a lack of comparative data, choice of skin incision is left to operator judgment, although many employ vertical incisions for better access and visualization whereas both the FIGO and SOGC guidelines recommend midline skin incision sufficiently high to allow a hysterotomy above the superior placental margin in high-suspicion PAS with major anterior previa. The ACOG/SMFM and FIGO guidelines indicate that in most cases when hysterectomy is necessary, a total hysterectomy is required because lower uterine segment or cervical bleeding due to cervical involvement frequently precludes a supra-cervical (partial) hysterectomy, Total hysterectomy also reduces the potential risk of malignancy developing in the cervical stump, the need for regular cervical cytology and other associated problems such as bleeding or discharge.

\section{UNRESOLVED ISSUES}


All authors and experts involved in developing the different guidelines noted that most current information on both prenatal diagnosis and management is derived from retrospective cohort studies without controls, case series and expert opinion. A search of PubMed using the MeSH headings for "placenta accreta, placenta increta, placenta percreta, abnormally invasive placenta and morbidly adherent placenta" published between May 2019 and May 2020 found 189 articles including 122 retrospective and two prospective cohort studies, 30 cases reports, 22 expert opinions, 7 case series, five systematic review and meta-analyses and only one RCT [54]. Furthermore, the absence of detailed histopathological evidence and lack of standardized clinical or photographic evidence of PAS at birth in around $90 \%$ of cohort studies $[11,12]$ considerably limits the value of the corresponding data and make it is impossible to reproduce such results in other centers or populations, or carry out meaningful meta-analysis. This highlights the difficulties of finding high quality evidence to support strong recommendations.

There are some generally agreed upon strategies, but comparison of the different guidelines shows (Table 5), that even those recommendations graded as strong, in particular regarding management, are not supported by well-performed RCTs. Interestingly, both the FIGO and SOGC strongly recommend the use of tranexamic acid during surgery for PAS but there are no cohort series available in the literature and only one small RCTs in cases of (non-accreta) placenta previa combining bilateral uterine artery ligation with tranexamic acid [55].

The pivotal role of ultrasound imaging in the screening women at risk and in accurately diagnosing PAS prenatally is highlighted in all guidelines. However, the ACOG/SMFM guidelines indicate that the standardized description of ultrasonography features of PAS [17] and pro forma for standardized reporting of 
ultrasound [18] proposed by an international group of imaging experts are not yet in widespread use in the U.S. and there is no evidence that they are routinely used in many other countries. The diagnosis of PAS is not part of general ultrasound training courses in the U.K [56] and it has only been added to the fetal medicine foundation website earlier this year (www//coursesf.etalmedicine.com). However, such ultrasound training and screening programs have existed for more than two decades for the detection of fetal anomalies such as congenital heart defect. Surprisingly, as this was one of the first objective of the use of ultrasound in obstetrics, the criteria used by authors of cohort series and gestational age at confirmation diagnosis of placenta previa remains highly variable [12]. Considering the increasing incidence of placenta previa accreta around the world and the high maternal morbidity and mortality at delivery of undiagnosed cases, similar international screening protocols with standard anatomical views for both low-lying/placenta previa and PAS should be implemented.

\section{Conflicts of interest}

The authors have no conflicts of interest. 


\section{SUMMARY (max 250 words)}

The changes in the epidemiology of placenta previa and placenta accreta spectrum (PAS) have been closely linked to the rapid rise in caesarean delivery (CD) rates over the last three decades. From a rare pathologic condition, PAS has become a new risk factor for major obstetric complications. The changes in the prevalence of PAS lag behind the rise in CDs by around 6 years and health providers must prepare for a significant rise in the numbers of pregnancies complicated by placenta previa accreta. New national and international guidelines or the update of existing ones for the diagnosis and management of PAS have highlighted the importance of the early identification of women with the condition. This is especially true for those presenting with an anterior low-lying/placenta praevia and one or more prior CD since they are at the highest risk of PAS. The crucial role of ultrasound imaging for the antenatal screening and diagnosis, the need for women diagnosed with PAS to be cared for by a multidisciplinary team and the importance of avoiding the placenta during the hysterotomy and/or attempt to remove it when PAS is suspected at delivery are key to reducing morbidity. Most current information on both prenatal diagnosis and management is derived from uncontrolled retrospective cohort studies and case series; in around $90 \%$ of cases there is no detailed histopathological evidence to confirm the severity of the PAS encountered. All guidelines agree on the need for new prospective controlled cohort studies and randomized controlled trials to compare the efficacy of the different therapeutic approaches and the use additional management techniques. 


\section{PRACTICE POINTS}

- Women presenting with low-lying/placenta previa and a history of one or more CDs should be carefully assessed for PAS.

- The diagnosis of PAS should be confirmed by trained operators in ultrasound imaging and regular follow-up should be arranged until delivery.

- $M R I$ is not essential for the diagnosis of PAS and its used is limited by cost and the availability of radiology experts in the evaluation of PAS

- Delivery should be planned and managed by an MDT with regular established expertise in complex uterine surgery and access to blood products and adult and neonatal ICUs.

- The optimal time of delivery is guided by maternal symptoms associated with placenta previa and must balance the risks and advantages for mother and baby which may be different in low- median- and high-income countries.

\section{RESEARCH AGENDA}

Health provision for the development of MDT with specialist surgeons, equipment, drugs, blood bank and intensive care infrastructure to safely manage women presenting with PAS requires an accurate evaluation of its prevalence and outcome which entails the use of standardized antenatal and post-natal diagnostic protocols. Within this context, there is a need for prospective multi-centre studies with participatory methodologies involving local service providers and management adapted to the grade of PAS and local facilities. There is also a need to evaluate the health and economic consequences of high caesarean section rates on maternal health within particular population context. 


\section{REFERENCES}

1. Polak JO, Phelan GW. Placenta accreta; incidence, pathology, and management, Surg Gynec Obst 1924;38:1815.

2. Andrews CJ. Report of a case of retained placenta, clinically placenta accreta. JAMA. 1924;82:1780.

3. Forster DS. A case of placenta accreta. Can Med Assoc J. 1927;17:204-7.

4. Nathanson JL. The anatomy, genesis and clinical considerations of placenta accreta. Am J Obstet Gynecol. 1928;16:44-50.

5. Irving C, Hertig AT. A study of placenta accreta. Surg Gynecol Obstet 1937;64:178-200.

6. Gielchinsky Y, Rojansky N, Fasouliotis SJ, Ezra Y. Placenta accreta-summary of 10 years: a survey of 310 cases. Placenta. 2002;23:210-4.

7. Sheiner E, Levy A, Katz M, Mazor M. Identifying risk factors for peripartum cesarean hysterectomy. A population-based study. J Reprod Med. 2003;48:622-6.

8. Bencaiova G, Burkhardt T, Beinder E. Abnormal placental invasion experience at 1 center. J Reprod Med. 2007;52:709-14.

9. Woodring TC, Klauser CK, Bofill JA, Martin RW, Morrison JC. Prediction of placenta accreta by ultrasonography and color Doppler imaging. J Matern Fetal Neonatal Med. 2011;24:118-21.

10. Klar M, Laub M, Schulte-Moenting J, Proempeler H, Kunze M. Clinical risk factors for complete and partial placental retention: a case-control study. J Perinat Med. 2014;41:529-34.

11. Jauniaux E, Bunce C, Grønbeck L, Langhoff-Roos J. Prevalence and main outcomes of placenta accreta spectrum: a systematic review and metaanalysis. Am J Obstet Gynecol. 2019;9:e031193.

12. Jauniaux E, Grønbeck L, Bunce C, Langhoff-Roos J, Collins SL. Epidemiology of placenta previa accreta: a systematic review and metaanalysis. BMJ Open. 2019;9:e031193.

13. * Luke RK, Sharpe JW, Greene RR. Placenta accreta: The adherent or invasive placenta. Am J Obstet Gynecol 1966;95:660-8.

14. Jauniaux E, Ayres-de-Campos D, Langhoff-Roos J, Fox KA, Collins S; FIGO Placenta Accreta Diagnosis and Management Expert Consensus Panel. FIGO 
classification for the clinical diagnosis of placenta accreta spectrum disorders. Int J Gynaecol Obstet. 2019;146:20-24.

15. Jauniaux E, Hussein AM, Fox KA, Collins SL. New evidence-based diagnostic and management strategies for placenta accreta spectrum disorders. Best Pract Res Clin Obstet Gynaecol. 2019;61:75-88.

16. Hecht JL, Baergen R, Ernst LM, Katzman PJ, Jacques SM, Jauniaux E et al. Classification and reporting guidelines for the pathology diagnosis of placenta accreta spectrum (PAS) disorders: recommendation from an expert panel. Modern Pathol 2020; in press.

17. * Collins SL, Ashcroft A, Braun T, Calda P, Langhoff-Ross J, Morel O et al., Proposed for standardized ultrasound descriptions of abnormally invasive placenta (AIP). Ultrasound Obstet Gynecol. 2016;47:271-275.

18. Alfirevic Z, Tang A-W, Collins SL, Robson SC, Palacios-Jaraquemadas, on behalf of the Ad-hoc International AIP Expert group. Pro forma for ultrasound reporting in suspected abnormally invasive placenta (AIP); an international consensus. Ultrasound Obstet Gynecol. 2016;47:276-278.

19. Morel O, Collins SL, Uzan-Augui J, Masselli G, Duan J, Chabot-Lecoanet AC, et al; International Society for Abnormally Invasive Placenta (IS-AIP). A proposal for standardized magnetic resonance imaging (MRI) descriptors of abnormally invasive placenta (AIP) - From the International Society for AIP.Diagn Interv Imaging 2019;100:319-325.

20. Jauniaux E, Silver RM, Matsubara S. The new world of placenta accreta spectrum disorders. Int J Gynaecol Obstet. 2018;140:259-260.

21. Silver RM, Landon MB, Rouse DJ, Leveno KJ, Spong CY, Thom EA, et al. National Institute of Child Health and Human Development Maternal-Fetal Medicine Units Network. Maternal morbidity associated with multiple repeat cesarean deliveries. Obstet Gynecol. 2006;107:1226-32.

22. *Jauniaux E, Chantraine F, Silver RM, Langhoff-Roos J; FIGO Placenta Accreta Diagnosis and Management Expert Consensus Panel. FIGO consensus guidelines on placenta accreta spectrum disorders: Epidemiology. Int J Gynaecol Obstet. 2018;140:265-273.

23. Gurol-Urganci I, Cromwell DA, Edozien LC, et al. Risk of placenta previa in second birth after first birth cesarean section: a population-based study and meta-analysis. BMC Pregnancy Childbirth. 2011;11:95. 
24. Fitzpatrick KE, Sellers S, Spark P, Kurinczuk JJ, Brocklehurst P, Knight M. Incidence and risk factors for placenta accreta/increta/percreta in the UK: a national case-control study. PLoS One. 2012;7:e52893.

25. Jauniaux E, Jurkovic D. Placenta accreta: pathogenesis of a 20th century iatrogenic uterine disease. Placenta. 2012;33:244-51.

26. Jauniaux E, Bhide A, Burton GJ. Pathophysiology of accreta. In: Silver R, ed. Placenta accreta syndrome. Portland: CRC Press, 2017:13-28.

27. Zosmer N, Fuller J, Shaikh H, Johns J, Ross JA. Natural history of early firsttrimester pregnancies implanted in Cesarean scars. Ultrasound Obstet Gynecol. 2015;46:367-75.

28. Calì G, Timor-Tritsch IE, Palacios-Jaraquemada J, Monteaugudo A, Buca D, Forlani F, et al. Outcome of Cesarean scar pregnancy managed expectantly: systematic review and meta-analysis. Ultrasound Obstet Gynecol. 2018;51:169-175.

29. Baldwin HJ, Patterson JA, Nippita TA, Torvaldsen S, Ibiebele I, Simpson JM, Ford JB. Antecedents of abnormally invasive placenta in primiparous women: risk associated with gynecologic procedures. Obstet Gynecol. 2018;131:22733.

30. Gyamfi-Bannerman C, Gilbert S, Landon MB, Spong CY, Rouse DJ, Varner MW et al; Eunice Kennedy Shriver National Institute of Child Health and Human Development (NICHD) Maternal-Fetal Medicine Units (MFMU) Network. Risk of uterine rupture and placenta accreta with prior uterine surgery outside of the lower segment. Obstet Gynecol. 2012;120:1332-7.

31. Jauniaux E, Moffett A, Burton GJ. Placental Implantation Disorders. Obstet Gynecol Clin North Am. 2020;47:117-132.

32. Jauniaux E, Hussein AM, Zosmer N, Elbarmelgy RM, Elbarmelgy RA, Shaikh $H$, Burton GJ. A new methodologic approach for clinico-pathologic correlations in invasive placenta previa accreta. Am J Obstet Gynecol. 2020; 222:379.e1-379.e11369.

33. Jauniaux E, Grobmann W. Caesarean section: The world No 1 operation. A textbook of caesarean section. Edts: Jauniaux E, Grobman W. Oxford University Press. Oxford. 1-8, 2016.

34. Appropriate technology for birth. Lancet 1985;2:436-7. 
35. Boerma T, Ronsmans C, Melesse DY, Barros AJD, Barros FC, Juan L, et al. Global epidemiology of use of and disparities in caesarean sections. Lancet. 2018;392:1341-1348.

36. Solheim KN, Esakoff TF, Little SE, Cheng YW, Sparks TN, Caughey AB. The effect of cesarean delivery rates on the future incidence of placenta previa, placenta accreta, and maternal mortality. J Matern Fetal Neonatal Med. 2011;24:1341-6.

37. Knight M, Nair M, Tuffnell D, Shakespeare J, Kenyon S, Kurinczuk JJ (Eds.) on behalf of MBRRACE-UK. Saving Lives, Improving Mothers' Care Lessons learned to inform maternity care from the UK and Ireland Confidential Enquiries into Maternal Deaths and Morbidity 2013-15. Oxford: National Perinatal Epidemiology Unit, University of Oxford; 2017 [www.npeu.ox.ac. uk/mbrrace-uk].

38. * Jauniaux E, Alfirevic Z, Bhide AG, Belfort MA, Burton GJ, Collins SL et al; Royal College of Obstetricians and Gynaecologists. Placenta Praevia and Placenta Accreta: Diagnosis and Management: Green-top Guideline No. 27a. BJOG. 2019;126:e1-e48.

39. * Obstetric Care Consensus No. 7 Summary: Placenta Accreta Spectrum. Obstet Gynecol. 2018;132:1519-21.

40. Society of Gynecologic Oncology; American College of Obstetricians and Gynecologists and the Society for Maternal-Fetal Medicine, Cahill AG, Beigi R, Heine RP, Silver RM, Wax JR. Placenta Accreta Spectrum. Am J Obstet Gynecol. 2018;219:B2-B16.

41. Jauniaux E, Ayres-de-Campos D; FIGO Placenta Accreta Diagnosis and Management Expert Consensus Panel. FIGO consensus guidelines on placenta accreta spectrum disorders: Introduction. Int J Gynaecol Obstet. 2018;140:261-264.

42. Jauniaux E, Bhide A, Kennedy A, Woodward P, Hubinont C, Collins S; FIGO Placenta Accreta Diagnosis and Management Expert Consensus Panel. FIGO consensus guidelines on placenta accreta spectrum disorders: Prenatal diagnosis and screening. Int J Gynaecol Obstet. 2018;140:274-280.

43. Allen L, Jauniaux E, Hobson S, Papillon-Smith J, Belfort MA; FIGO Placenta Accreta Diagnosis and Management Expert Consensus Panel. FIGO 
consensus guidelines on placenta accreta spectrum disorders: Nonconservative surgical management. Int J Gynaecol Obstet. 2018;140:281-90.

44. Sentilhes L, Kayem G, Chandraharan E, Palacios-Jaraquemada J, Jauniaux E; FIGO Placenta Accreta Diagnosis and Management Expert Consensus Panel. FIGO consensus guidelines on placenta accreta spectrum disorders: Conservative management. Int J Gynaecol Obstet. 2018;140:291-298.

45. Hobson SR, Kingdom JC, Murji A, Windrim RC, Carvalho JCA, Singh SS, et al. No. 383-Screening, Diagnosis, and Management of Placenta Accreta Spectrum Disorders. J Obstet Gynaecol Can. 2019;41:1035-49.

46. Cal M, Ayres-de-Campos D, Jauniaux E. International survey of practices used in the diagnosis and management of placenta accreta spectrum disorders. Int J Gynaecol Obstet. 2018;140:307-311.

47. Sargent W, Collins SL. Are women antenatally diagnosed with abnormally invasive placenta receiving optimal management in England? An observational study of planned place of delivery. Acta Obstet Gynecol Scand. 2019;98:337-41.

48. Bartels HC, Rogers AC, O'Brien D, McVey R, Walsh J, Brennan DJ. Association of Implementing a multidisciplinary team approach in the management of morbidly adherent placenta with maternal morbidity and mortality. Obstet Gynecol. 2018;132:1167-76.

49. Shamshirsaz AA, Fox KA, Salmanian B, Diaz-Arrastia CR, Lee W, Baker BW, et al. Maternal morbidity in patients with morbidly adherent placenta treated with and without a standardized multidisciplinary approach. Am J Obstet Gynecol. 2015;212:218.e1-9.

50. Shamshirsaz AA, Fox KA, Erfani H, Clark SL, Salmanian B, Baker BW et al., Multidisciplinary team learning in the management of the morbidly adherent placenta: outcome improvements over time. Am J Obstet Gynecol. 2017;216:612.e1-612.e5.

51. Esakoff TF, Handler SJ, Granados JM, Caughey AB. PAMUS: placenta accreta management across the United States. J Matern Fetal Neonatal Med. 2012;25:761-5.

52. Wright JD, Silver RM, Bonanno C, Gaddipati S, Lu YS, Simpson LL, et al. Practice patterns and knowledge of obstetricians and gynecologists regarding placenta accreta. J Matern Fetal Neonatal Med. 2013;26:1602-9. 
53. loscovich A, Shatalin D, Butwick AJ, Ginosar Y, Orbach-Zinger S, Weiniger CF. Israeli survey of anesthesia practice related to placenta previa and accreta. Acta Anaesthesiol Scand. 2016;60:457-64.

54. Hussein AM, Dakhly DMR, Raslan AN, Kamel A, Abdel Hafeez A, Moussa M, et al. The role of prophylactic internal iliac artery ligation in abnormally invasive placenta undergoing caesarean hysterectomy: a randomized control trial. J Matern Fetal Neonatal Med. 2019;32:3386-92.

55. Abbas AM, Shady NW, Sallam HF. Bilateral uterine artery ligation plus intravenous tranexamic acid during cesarean delivery for placenta previa: a randomized double-blind controlled trial. J Gynecol Obstet Hum Reprod. 2019;48:115-9.

56. National Collaborating Centre for Womens and Children's Health. Antenatal care: routine care for the healthy pregnant woman. Clinical Guideline. London: RCOG Press; 2010. 
Table 1: Summary of epidemiology data and distribution of PAS grades in population studies [11] and studies of placenta previa accreta [12] diagnosed antenatally.

\begin{tabular}{|l|l|l|}
\multicolumn{2}{c}{ Population } \\
\begin{tabular}{|l|l|} 
No of articles included in \\
study
\end{tabular} & 29 & 20 \\
\hline Retrospective/Prospective & $22 / 7$ & $13 / 7$ \\
\hline $\begin{array}{l}\text { No of cases of PAS/births \& } \\
\text { pregnancies (Prevalence) }\end{array}$ & $\begin{array}{l}7,001 / 5,719,992 \\
(0.12 \%) \\
1 / 817\end{array}$ & $\begin{array}{l}587 / 1,231,160(0.05 \%) \\
1 / 2,097\end{array}$ \\
\hline $\begin{array}{l}\text { No of PAS cases with } \\
\text { detailed histopathology/total } \\
\text { No of cases in study (\%) }\end{array}$ & $\begin{array}{l}770 / 832 \\
(92.5 \%)\end{array}$ & $\begin{array}{l}283 / 309 \\
(91.6 \%)\end{array}$ \\
\hline Placenta creta/adherenta & $473(61.4 \%)$ & $171(60.4 \%)$ \\
& Range 34.8-81.6\% & Range $30.4-92.3 \%$ \\
\hline Placenta increta & $126(16.4 \%)$ & $74(26.2 \%)$ \\
& Range 3.7-43.5\% & Range $0-45.7 \%$ \\
\hline Placenta percreta & $171(22.2 \%)$ & $38(13.4 \%)$ \\
& Range $6.6-51.9 \%$ & Range $2.4-35.3 \%$ \\
\hline
\end{tabular}

PAS $=$ Placenta Accreta Spectrum 
Table 2. RCOG

1++ High-quality meta-analyses, systematic reviews of randomised controlled trials or randomised controlled trials with a very low risk of bias

1+ Well-conducted meta-analyses, systematic reviews of randomised controlled trials or randomised controlled trials with a low risk of bias

1- Meta-analyses, systematic reviews of randomised controlled trials or randomised controlled trials with a high risk of bias

2++ High-quality systematic reviews of case-control or cohort studies or high-quality case-control or cohort studies with a very low risk of confounding, bias or chance and a high probability that the relationship is causal

$2+\quad$ Well-conducted case-control or cohort studies with a low risk of confounding, bias or chance and a moderate probability that the relationship is causal

2- $\quad$ Case-control or cohort studies with a high risk of confounding, bias or chance and a significant risk that the relationship is not causal

$3 \quad$ Non-analytical studies, e.g. case reports, case series

$4 \quad$ Expert opinion

\section{Grades of Recommendation}

A At least one meta-analysis, systematic reviews or RCT rated as 1++, and directly applicable to the target population; or a systematic review of RCTs or a body of evidence consisting principally of studies rated as $1+$, directly applicable to the target population and demonstrating overall consistency of results

B A body of evidence including studies rated as 2++ directly applicable to the target population, and demonstrating overall consistency of results; or Extrapolated evidence from studies rated as $1++$ or $1+$

C A body of evidence including studies rated as 2+ directly applicable to the target population, and demonstrating overall consistency of results; or Extrapolated evidence from studies rated as $2++$

D Evidence level 3 or 4; or Extrapolated evidence from studies rated as $2+$

Good Practice Points (GPP) Recommended best practice based on the clinical experience of the guideline development group 
Table 3. ACOC/SMFM

Grading of recommendations assessment

1A. Strong recommendation, high-quality evidence

1B. Strong recommendation, moderate-quality evidence

. Strong recommendation,

low-quality evidence

high-quality evidence

2B. Weak recommendation, moderate-quality evidence

2C. Weak recommendation, low-quality evidence

Best practice

$\mathrm{RCT}=$ randomized controlled trial

\section{Quality of supporting evidence}

Consistent evidence from well performed RCTs or overwhelming evidence of some other form. Further research is unlikely to change confidence in the estimate of benefit and risk. Evidence from RCTs with important limitations (inconsistent results, methodologic flaws, indirect or imprecise), or very strong evidence of some other research design. Further research (if performed) is likely to have an impact on confidence in the estimate of benefit and risk and may change the estimate.

Evidence from observational studies, unsystematic clinical experience, or from RCTs with serious flaws. Any estimate of effect is uncertain.

Consistent evidence from well performed RCTs or overwhelming evidence of some other form. Further research is unlikely to change confidence in the estimate of benefit and risk. Evidence from RCTs with important limitations (inconsistent results, methodologic flaws, indirect or imprecise), or very strong evidence of some other research design. Further research (if performed) is likely to have an effect on confidence in the estimate of benefit and risk and may change the estimate. Evidence from observational studies, unsystematic clinical experience, or from RCTs with serious flaws. Any estimate of effect is uncertain.

Recommendation in which either (i) there is enormous amount of indirect evidence that clearly justifies strong recommendation (direct evidence would be challenging, and inefficient use of time and resources, to bring together and carefully summarize), or (ii) recommendation to contrary would be unethical. 
Table 4. SOGC Key to evidence statements and grading of recommendations

\section{Quality of evidence assessment}

I: Evidence obtained from at least one properly
RCT.

II-1: Evidence from well-designed controlled trials without randomization.

II-2: Evidence from well-designed cohort (prospective or retrospective) or case-control studies, preferably from more than one centre or research group.

II-3: Evidence obtained from comparisons between times or places with or without the intervention. Dramatic results in uncontrolled experiments (such as the results of treatment with penicillin in the 1940s) could also be included in this category

III: Opinions of respected authorities, based on clinical experience, descriptive studies, or reports of expert committees

$\mathrm{RCT}=$ randomized controlled trial.

\section{Classification of recommendations}

A. There is good evidence to recommend the clinical preventive action.

B. There is fair evidence to recommend the clinical preventive action.

C. The existing evidence is conflicting and does not allow to make a recommendation for or against use of the clinical preventive action; however, other factors may influence decisionmaking.

D. There is fair evidence to recommend against the clinical preventive action.

E. There is good evidence to recommend against the clinical preventive action.

L. There is insufficient evidence (in quantity or quality) to make a recommendation; however, other factors may influence decision making. 


\section{PRACTICE POINTS}

- Women presenting with low-lying/placenta previa and a history of one or more CDs should be carefully assessed for PAS.

- The diagnosis of PAS should be confirmed by trained operators in ultrasound imaging and regular follow-up should be arranged until delivery.

- MRI is not essential for the diagnosis of PAS and its used is limited by cost and the availability of radiology experts in the evaluation of PAS

- Delivery should be planned and managed by an MDT with regular established expertise in complex uterine surgery and access to blood products and adult and neonatal ICUs.

- The optimal time of delivery is guided by maternal symptoms associated with placenta previa and must balance the risks and advantages for mother and baby which may be different in low- median- and high-income countries. 


\section{RESEARCH AGENDA}

Health provision for the development of MDT with specialist surgeons, equipment, drugs, blood bank and intensive care infrastructure to safely manage women presenting with PAS requires an accurate evaluation of its prevalence and outcome which entails the use of standardized antenatal and post-natal diagnostic protocols. Within this context, there is a need for prospective multi-centre studies with participatory methodologies involving local service providers and management adapted to the grade of PAS and local facilities. There is also a need to evaluate the health and economic consequences of high caesarean section rates on maternal health within particular population context. 


\section{MCQs for the manuscript: A comparison of recent guidelines in the diagnostic and management of placenta accreta spectrum disorders}

1. The following is true about the epidemiology of PAS:

a. Less than $30 \%$ of women diagnosed prenatally with PAS present with a low-lying/placenta previa and a surgical history of at least one prior uterine surgery, most commonly cesarean delivery (CD).

b. The incidence of placenta previa accreta increases with the number of prior CDs.

c. Prior gynecologic surgery (myomectomy, uterine adhesiolysis, septal resection) and some uterine pathology such as bicornuate uterus have not been associated with the development of PAS in subsequent pregnancies.

d. Large cesarean scar defects (CSD) can lead to the development of cesarean scar pregnancies (CSP) which can be a precursor for the development of PAS

$a=F ; b=T ; c=F ; d=T$

Explanation to the answers for Question 1:

a. CD rates have risen worldwide over the last decades sometime above $40 \%$ in countries such as Brazil, Turkey, Italy, Egypt, Argentina, Iran, South Korea and Mexico. As CD scar increase the risk of both previa and accreta placentation Today, over $95 \%$ of women diagnosed prenatally with PAS present with a low-lying/placenta previa and a surgical history of at least one prior CD.

b. Multiple CDs create substantial and chronic damage to the integrity of the uterine wall, especially if the endometrium fails to re-epithelize in an area of scar formation facilitating the development of PAS.

c. Accreta placentation has been described after most minor gynaecologic surgical procedure including myomectomy but the risk is low unless the uterine cavity is entered or the myometrium is perforated.

d. The diagnosis of CSP in the first trimester is increasingly common, such that the natural history of evolution to PAS has now been clearly established, especially for type-2 CSP. 
2. On the prenatal diagnosis of PAS the RCOG, ACOG/SMFM, FIGO \& SOGC agree that:

a. Antenatal diagnosis of PAS is crucial in planning its management and has been shown to reduce maternal morbidity and mortality.

b. Previous uterine curettages and the presence of an anterior lowlying/placenta praevia should alert the antenatal care team of the higher risk of PAS.

c. Ultrasonography is a relatively inexpensive and widely available imaging modality and therefore should be the first line for the diagnosis of PAS.

d. MRI is essential for making a prenatal diagnosis of suspected PAS and should be performed in all cases of women at risk of PAS.

$\mathrm{a}=\mathrm{T} ; \mathrm{b}=\mathrm{F} ; \mathrm{c}=\mathrm{T} ; \mathrm{d}=\mathrm{F}$

Explanation to the answers for Question 2:

a. There is mounting evidence that women with PAS diagnosed prenatally and managed by a multidisciplinary team (MDT) in a centre of excellence (CoE) are less likely to require emergency surgery, large-volume blood transfusion and reoperation within 7 days of delivery for bleeding complications compared with women managed by standard obstetric care without a specific protocol.

b. Uterine curettage in particular if complicated with uterine perforation usually damage the myometrium above the lower segment. These can be associated with focal PAS of the anterior uterine wall or fundus but the patients with the highest risk of PAS are those with prior multiple CDs presenting with an anterior low-lying/placenta previa.

c. Ultrasound is operator dependent and until recently there was no training available for the ultrasound diagnosis of PAS but in expert hands it has a positive predictive value, negative predictive value and detection rate above $95 \%$. The diagnosis of PAS is not part of general ultrasound training courses in the U.K and it has only been added to the fetal medicine foundation website earlier this year (www//coursesf.etalmedicine.com).

d. MRI may be useful in evaluating the pelvic extension of a placenta percreta or areas difficult to evaluate on ultrasound, but not essential and its effectiveness is currently limited by the relative contraindication to the use of a gadolinium contrast enhancing agent. 
3. On preparation for delivery and management of PAS the RCOG, ACOG/SMFM, FIGO \& SOGC recommend that:

a. Women diagnosed with PAS can be cared for by obstetricians with experience in complex CDs in a local hospital close to the patient home.

b. Antenatal corticosteroids should be given for lung maturation at 30 weeks for planned delivery at 36-37 weeks of gestation.

c. No attempt should be made to remove the placenta (extirpative approach or forcible manual removal of the placenta) if it shows no signs of separation as this may cause substantial hemorrhage.

d. Conservative management or expectant management with adjuvant therapy such as methotrexate (MTX) should be considered in all cases of PAS.

$a=F ; b=F ; c=T ; d=F$

Explanation to the answers for Question 3:

a. Patients diagnosed with PAS should be cared for by a MDT with expertise in complex pelvic surgery in a specialist centre with logistic support for immediate access to blood products, adult and neonatal intensive care, using a preoperative coordination with protocol-based interdisciplinary care including anesthesiology, hematology/blood bank, maternal-fetal medicine, neonatology, and expert pelvic surgeons to optimize intraoperative and post-operative outcomes.

b. As the timing of delivery decisions needs to balance maternal risks and benefits with those of the fetus or neonate and most patients presents with a placenta previa accreta antenatal, corticosteroids should be used for lung maturation following the international gestational age-based recommendations for anticipated delivery before term. Here the guidelines differ regarding the timing of delivery with the ACOG/SMFM recommending $34^{+0}-35^{+6}$ weeks of gestation for scheduled cesarean delivery in a stable patient whereas for the RCOG, recommends delivery at $35^{+0}-36^{+6}$ weeks of gestation in the absence of risk factors for preterm delivery

c. Attempt at removing the placenta at delivery in case of PAS is inevitably associated with massive obstetric hemorrhage and a high maternal morbidity and some maternal mortality. If at the time of an elective repeat caesarean section, PAS is suspected based on uterine appearance and there are no extenuating circumstances mandating immediate delivery, the caesarean section should be delayed until until optimal surgical expertise arrives and adequate blood products are available rather than attempting at delivering the placenta.

d. Conservative management or expectant management should be considered only for carefully selected cases of PAS after detailed counseling about the risks, uncertain benefits, and efficacy and local arrangements need to be made to ensure regular review, ultrasound examination and access to emergency care should the patient experience complications. MTX adjuvant therapy should not be used for expectant 
management as it is of unproven benefit and has significant adverse effects, including maternal mortality. 